\title{
Charles William Wendte als interreligiöser Brückenbauer
}

\author{
Michael Sturm-Berger
}

\section{Unitarier beim Weltkongress in Berlin 1910}

Das Thema dieser Tagung beschäftigt mich schon seit einiger Zeit. Um es genau zu sagen: seit wir in Berlin die Jubiläums-Feier aus Anlass von 100 Jahren interreligiöse Konferenzen in Berlin und Deutscbland vorbereiteten. Diese erste größere Konferenz in Deutschland mit interreligiösen Anteilen war der Fünfte Weltkongress für freies Christentum und religiösen Fortschritt im Jahre 1910.

Zunächst hatten wir sehr mit der Organisation dieser Veranstaltung zu tun. Allmählich aber schälten sich bei Lektüre des 814 Seiten starken Kongressbandes immer mehr die Personalfragen heraus: Wer hatte diesen gewaltigen Kongress mit über zweitausend angemeldeten und mehreren tausend spontanen Teilnehmern überhaupt organisiert? - Die Hauptlast auf deutscher Seite scheinen der Protestantenverein und die „Freunde der christlichen Welt“ getragen zu haben, welche bis 1941 ihre eigenen Zeitschriften herausgaben, dann aber vom Nazi-Regime mundtot gemacht wurden.

Teilnehmer beim Berliner Weltkongress war übrigens auch Rudolf Walbaum (1869-1948), damals Prediger der Religionsgemeinschaft freier Protestanten in Rheinhessen (gegr. 1876), welcher in Alzey, südwestlich von Mainz, residierte. Er lernte beim Berliner Weltkongress die Unitarier aus den USA, Großbritannien und Ungarn bzw. Siebenbürgen kennen. 1911 fügte Walbaum seiner Zeitschrift Der Freiprotestant den Untertitel Deutsch-unitarische Blätter hinzu. Es gibt also eine Verbindung zwischen dem 1910er Kongress und den modernen Unitariern in Deutsch- 
land. Der nun als Thema gewählte Charles W. Wendte soll damals jedoch Bremen als „die unitarischste aller deutschen Städte“ "bezeichnet haben. ${ }^{1}$

Wer aber waren die damaligen Unitarier, jene Mitorganisatoren des Weltkongresses in Berlin? - Dessen internationaler General-Sekretär war der unitarische Pfarrer Dr. Charles William Wendte. Weshalb war es dazu gekommen, dass ein amerikanischer Unitarier die Ausrichtung eines religiösen Weltkongresses in Deutschland betrieb?

\section{Herkunft, Kindheit und Jugend C. W. Wendtes}

Zunächst erscheint bemerkenswert, wenn auch nicht außergewöhnlich, dass die Eltern von Charles W. Wendte aus Deutschland, aus dem damaligen Königreich Hannover stammten. Ihre Hochzeitsreise verbanden sie kurzerhand - vielleicht schon etwas ungewöhnlicher - im Herbst 1842 mit ihrer Auswanderung in die USA - per Segelschiff über Bremen und New York nach Boston.

Wir sind über diese Geschehnisse relativ gut informiert, weil Rev. Dr. Wendte im Alter von 83 Jahren eine über 1200-seitige Autobiografie in zwei Bänden veröffentlichte, die er The Wider Fellowship nannte, also etwa: „Die umfassende Gemeinschaft". Sie bemerken vermutlich schon meine Begeisterung für das Lebenswerk dieses Mannes - und das hat viele Gründe.

Doch kehren wir noch einmal kurz zu seinen Eltern zurück, welche beide protestantisch erzogen waren. Im Mai 1843 gingen sie in Boston spazieren, als sie eine Kirche mit offenen Türen und feierlicher Orgelmusik erreichten. ${ }^{2}$ Seine spätere Mutter fühlte sich hingezogen und sein späterer Vater schlug vor hineinzugehen. Während sie damals noch kaum Englisch verstand, hörte er gut zu und war von der Predigt entzückt, weil sie ihm so vernünftig erschien.

Sie können sich jetzt schon denken, dass es eine unitarische Kirche war, in welche die Eltern in spe - ob durch Zufall oder Fügung - hineingeraten waren. Jedenfalls ging das Paar immer wieder in diese Kirche zum Sonntagsgottesdienst. Als schließlich ein Jahr später ihr erster Sohn Charles William am 11. Juni 1844 in Boston geboren wurde, erklärte sein Vater Carl: „Wir werden ihn zu einem unitarischen Geistlichen machen.“"3

Aber für Wendte war der Weg nicht ganz so einfach, wie man denken könnte, denn sein Vater starb bereits im Jahre 1848, als seine Söhne knapp vier und zwei Jahre alt waren. Ihre junge, verwitwete Mutter Johanna arbeitete bald darauf als Lehrerin für deutsche Sprache und Literatur, um für Charles und seinen jüngeren Bruder William sorgen zu können. Im Frühling 1861 zog sie mit ihren Söhnen

\footnotetext{
1, Elke Schlinck-Lazarraga: Wiedergeburt schöpferischer Religion im Weltbund für religiöse Freibeit; I. Geschichte des Weltbundes. Norderstedt Juni 1975, S. 79.

2 C. W. Wendte: The Wider Fellowship. Memories, Friendships, and Endeavors for Religious Unity 1844-1927. 2 Bände. Boston, Mass. 1927, hier Bd. 1, S. 14f. - Alle englischsprachigen Texte wurden vom Autor selber übersetzt.

${ }^{3}$ Ebd., S. 16.
} 
nach San Francisco, wo Charles alsbald im dortigen Hafenamt eine Anstellung fand. Er lernte in San Francisco den unitarischen Pfarrer Thomas Starr King (1824-1864) kennen, welcher ihn offenkundig entscheidend darin beeinflusste, selber unitarischer Geistlicher zu werden, wie sein Vater es angekündigt hatte. Rev. T. S. King ist noch heute in den USA eine gewisse Berühmtheit, weil er im amerikanischen Bürgerkrieg für die Einheit des Staatenbundes eintrat, weshalb er als Nationalheld gilt und manchmal sogar als „Redner, welcher die Nation rettete“ bezeichnet wird.

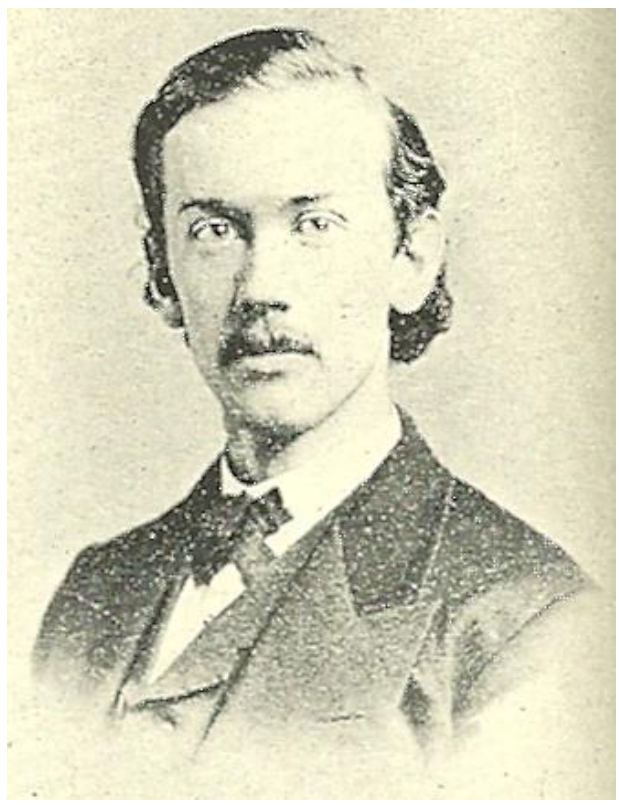

Abbildung 1: Charles W. Wendte 1868

(aus: The Wider Fellowship, Bd. 1, S. 192)

\section{Theologische Ausbildung und erste interreligiöse Eindrücke}

Seinen ersten größeren Überblick zu Entstehung und Wesen der Religion, zur Rolle von Vernunft und Gewissen erhielt Wendte nach eigener Aussage durch den aus Frankreich stammenden, ehemaligen katholischen Geistlichen Professor Charles F. B. Miel (1817-1902). Nachfolgend (1866-1869) studierte er an der Meadville Theological und der Harvard Divinity School in Boston unitarische Theologie. Bei dortigen Auseinandersetzungen zwischen konservativen und „radikalen“ Unitariern neigte Wendte deutlich den „Radikalen“ zu, stand deshalb zeitlebens Glaubensbekenntnissen skeptisch gegenüber. 
Rev. Dr. James Freeman Clarke (1810-1888), eine Gründerfigur seines Faches, machte ihn in der Vergleichenden Religionswissenschaft tiefer mit Judentum, klassisch-antiken Religionen, Brahmanismus, Islam und deren Beziehungen zur Lehre der Evangelien bekannt. Er vermittelte ihm dazu auch die Kunst der Selbstbeherrschung, wie Wendte später selber schrieb. So beschäftigte sich der Student bereits damals mit der Lehre des reform-hinduistischen Brabmo Samaj (gegründet 1828) und gegen Ende seines Studiums mit dem deutsch-schweizerischen Protestantenverein, welcher 1863 gegründet worden war. Er übersetzte nach eigenen Angaben dazu 1869 (?) einen Artikel für die Unitarier-Zeitschrift Christian Register aus dem Deutschen ins Englische.

Neben den unitarischen Kirchen verschiedener Länder sollten Brabmo Samaj und der Protestantenverein ihn noch viele Jahre bei seinen internationalen und interreligiösen Bestrebungen begleiten. Im Juni 1869 bestand er sein Examen und erhielt im selben Jahr eine Pfarrstelle in Chicago (Illinois). Dort beobachtete Wendte nach einem Großfeuer 1871 solidarische Aktionen der verschiedenen ReligionsGemeinschaften:

Aus der Mitte des Gedränges heraus kamen zwei Männer hervor, der eine ein wohlbekannter Priester der benachbarten katholischen Kirche, der andere der Rabbiner einer jüdischen Gemeinde. Mit ihrer Hilfe wurde die Aufgabe der Verteilung einfacher gemacht. Es war entzückend zu sehen, wie in dieser Stunde höchster Bedürftigkeit alle sektiererischen Differenzen vergessen waren. Der Priester hielt einen Schinken, von welchem der Rabbiner Scheiben für die hungrigen Armen abschnitt, ganz uneingedenk der alttestamentlichen Einschärfung des verbotenen Schweinefleisches, während die protestantischen Geistlichen fühlten, indem sie das Brot an ihre bedürftigen „Brüder“ austeilten, geweiht durch menschliche Liebe, dass es ein sakramentaler Akt war, dessen Wert keiner anzweifeln konnte. Unsere gemeinsame Katastrophe und Sorge machte uns alle einig in Glauben, Hoffnung und die Nächstenliebe ist die größte von allen. Es war eine schöne Prophezeiung einer besseren Zeit, die kommt, wenn religiöse Männer und Frauen sich über die abweichenden intellektuellen Meinungen und rituellen Bräuche erheben werden, welche nun trennen und sie oft erbittern, zu einer Anerkennung der gemeinsamen humanen Interessen der Menschheit; wenn alle vereinigt sein werden in einer großen Familie, Kinder des Allvaters. ${ }^{4}$

\footnotetext{
${ }^{4}$ Ebd., S. $260 f$.
} 


\section{Erste internationale Kontakte und „unitarische Mission“}

Das Pfarramt in Chicago strengte Wendte offenkundig an, und so machte er 1874 in einer Erschöpfungspause seine erste Europareise, zusammen mit seiner Mutter, wobei er auch versuchte, persönlichen Kontakt mit dem deutschen Protestantenverein aufzunehmen, welcher von britischen und ungarischen Unitariern bereits gepflegt wurde. Zurückgekehrt nach Chicago, nahm er u.a. mit reformjüdischen Kräften Verbindung auf und ließ von seiner Kanzel aus auch Frauen sprechen, da er von ihrem Verkündigungsrecht überzeugt war. In Chicago erschien sein erstes von vier (Kinder-) Gesangbüchern in hoher Auflage, woran man seine ausgeprägte Neigung zur Musik erkennen kann.

1875 siedelte Wendte aus gesundheitlichen Gründen nach Cincinnati (Ohio) über, wo er mit dem späteren US-Präsidenten William Howard Taft (1857-1930) zusammenarbeitete, welcher auch Unitarier war (1909-1913 amtierte er als USPräsident, 1921-1930 als oberster Richter der USA). Aus aktuellem Anlass sei bemerkt, dass Präsident Taft später scherzend bemerkt haben soll, Wendte habe „ihn ins Weiße Haus hineingebetet" ${ }^{\text {". }}$ Außerdem engagierte Wendte sich für seine afroamerikanischen Mitbürger. Danach (1882-1885) wirkte er in Newport (Rhode Island). Diese Zeit könnte wieder von besonderer Bedeutung gewesen sein, denn Rhode Island war der erste westliche Staat der Neuzeit, in welchem bereits in der Mitte des 17. Jahrhunderts der Baptisten-Prediger Roger Williams (1603-1683) vom britischen König Charles II. Religionsfreiheit erwirkt hatte. Dort ließ Wendte u.a. Protap Chunder Mozoomdar (Chóndro Mojumdar; 1840-1905) von seiner Kanzel predigen, den späteren Vertreter der Hindu-Vereinigung Brahmo Samaj beim Weltparlament der Religionen in Chicago. Anschließend (1886-1898) betrieb Wendte unitarische Mission an der amerikanischen Westküste (vorwiegend in Oakland und Los Angeles), nicht im Sinne der Verbreitung unitarischer Kirchenstrukturen, sondern unitarischer Gesinnung. Bereits im Herbst 1887 machte er die Bekanntschaft des unitarischen Theologie-Studenten Samuel Atkins Eliot II. (18621950), welcher nun zusammen mit ihm in diese Richtung wirkte und ein dauerhafter Weggefährte werden sollte.

\section{Eindrücke vom Weltparlament 1893 und Eheschließung}

Angesichts der oben geschilderten interreligiösen Zusammenarbeit nach dem Großfeuer in Chicago 1871, ist es vielleicht nicht verwunderlich, dass diese Stadt zweiundzwanzig Jahre später, im Rahmen der Weltausstellung 1893, zum Schauplatz des ersten Weltparlamentes der Religionen wurde. Wendte berichtete in seiner Autobiografie kurz über seine Teilnahme dort:

\footnotetext{
${ }^{5}$ Schlinck-Lazarraga: Wiedergeburt, S. 4.
} 
Die wundervolle Ausstellung von Genius und Industrie der Welt füllte mich mit Bewunderung und Entzücken. Das Parlament der Religionen, in welchem ich eine bescheidene Teilaufgabe übernahm, war ein packendes Experiment, indem es zum guten Teil einen lange gehegten Traum internationalen und inter-religiösen Einvernehmens erfüllte. Gleich nach meiner Rückkehr nach Hause redete ich über die Geschichte des Parlamentes der Religionen und seine dauerhaften Lehren für die religiöse Welt. Die einzige Kritik, die ich mir selber erlaubte, war, dass es eine Ausstellung war, indem sie mehr die gewaltige Reichweite und Mannigfaltigkeit von Religionen zeigte, als einen Ausdruck religiöser Sympathie, Einheit und Gemeinschaft. Das Spätere musste die vollere Entwicklung religiöser Kenntnis erwarten, interrassische Freundschaft und inter-religiöse Gutwilligkeit, welche die zukünftige Geschichte der Menschheit sicherlich bringen würden. ${ }^{6}$

Am 28. April 1896 heiratete Wendte in Oakland, fast 52-jährig, Abbie Louise Grant (1857-1936; Tochter des unitarischen Kaufmannes George Emery Grant in Oakland und dessen Gattin Ellen Louisa, geb. Dagett) - ein Schritt, den er später als sehr segensreich beschrieb. Die Gräber der Familien Grant und Wendte befinden sich noch heute auf dem Mountain View Cemetery in Oakland. Mittlerweile liegen mir - als ein erfreuliches Ergebnis dieses Göttinger Kongresses - Fotos von Wendtes Grabstätte vor, die er mit seiner Gattin teilt.

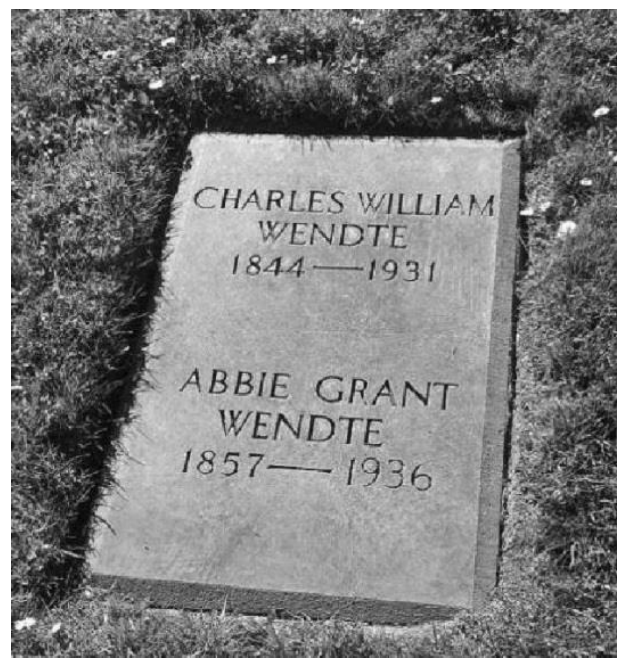

Abbildung 2: Grabplatte des Ehepaares Wendte auf dem Mountain View Cemetery (Bergblick-Friedhof) in Oakland/Kalifornien (Aufnahme vom 26.2.2013, mit freundlicher Genehmigung zur Veröffentlichung von Frau Dr. h.c. Arliss Ungar)

${ }^{6}$ Wendte: Fellowship, Bd. 2, S. 124. 


\section{Die Gründung einer internationalen und interreligiösen Organisation}

1899 reiste Wendte zum fünften Mal nach Europa; diesmal auf Wunsch seines Freundes Rev. Dr. Samuel A. Eliot, welcher mittlerweile Sekretär des amerikanischen Unitarierverbandes (AUA) war, um dessen 75-Jahr-Feier international zu gestalten. Zu jener Feier in Boston im Mai 1900 waren dann Vertreter von mindestens zehn verschiedenen liberalen Gemeinschaften anwesend, darunter Brahmo Samaj und der Protestantenverein. Bei diesem Anlass wurde der Internationale Rat von unitarischen und anderen liberal-religiös Denkenden und Wirkenden gegründet - mit Wendte als internationalem Generalsekretär. Man wollte weltweit zu denen Verbindung aufnehmen, welche danach strebten, reine Religion und vollkommene Freiheit zu vereinigen, um ihre Gemeinschaft und Zusammenarbeit zu fördern. Auf weitere Satzungen wurde verzichtet, aber der fünfzehnköpfige Vorstand sollte alle zwei bis drei Jahre einen Weltkongress in verschiedenen Ländern initiieren, was 1901-1913 tatsächlich gelang: Nur der vierte Kongress fand in den USA statt (Boston 1907), die fünf anderen in Europa, weshalb Wendte immer wieder Reisen dorthin unternahm.

Damit war meines Erachtens ein entscheidender Durchbruch erreicht: Der interreligiöse Dialog wurde erstmals für längere Zeit weltweit institutionalisiert - eine Qualität, die es zuvor niemals gegeben hatte - ein neues Zeitalter für die Religionen auf diesem Planeten hatte begonnen. Und sein Hauptaktivist sollte für die ersten zwanzig Jahre Charles Wendte sein. Er schrieb darüber wie folgt:

Ich habe die Geschichte dieses internationalen Unternehmens in solchem Detail aufgezeichnet, da sie ja von Bedeutung für die Ausbreitung liberaler Prinzipien war und da meine eigenen persönlichen Bemühungen von nun an eng mit ihr verbunden waren. Als ihr Sekretär eröffnete ich unverzüglich eine breite Korrespondenz mit Repräsentanten liberaler religiöser Gedanken überall in der Welt, indem ich ihnen einen Rundbrief zusandte, der die Prinzipien und Ziele des Rates verkörperte und um ihre Kooperation bat. Die erhaltenen Antworten waren fast einhellig zugeneigt und drückten die Freude der Schreiber aus, dass solch eine internationale und interreligiöse Bewegung eingeführt worden war. ${ }^{7}$

\section{Wendtes Bericht beim ersten Weltkongress in London 1901}

Die Zeit schien also reif für solch ein umfassendes Projekt, wenn es auch, wie wir wissen, später erhebliche Rückschläge geben sollte. Bereits beim ersten Weltkongress in London 1901 trug Wendte einen ausführlichen Bericht über die Entwicklung des Weltkongress-Projektes vor. Darin äußerte er auch Folgendes:

\footnotetext{
${ }^{7}$ Ebd., S. 193.
} 
Viele der empfangenen Briefe enthüllten die Einsamkeit und Bedrängnis, die von isolierten liberalen Denkern und Gemeinden rund um die Welt ertragen werden, ihr heroisches Zeugnis für Wahrheit und Freiheit und ihre Freude über die Gelegenheit, die ihnen von diesem Rat geboten wurde, in geschwisterliche Beziehungen zu ihren Mit-Liberalen in Europa und Amerika zu kommen. [...] Der bemerkenswerte Fortschritt in Zivilisierung während der vergangenen Jahrhunderte brachte die Nationen der Erde in engere und komplexere Beziehungen und machte ihnen, wie niemals zuvor, ihre gegenseitigen Abhängigkeiten und gegenseitigen Verpflichtungen bewusst. Die letztliche ,Föderation der Welt ${ }^{`}$ für ideale und geschwisterliche Ziele ist nicht länger bloß ein Poetentraum; sie ist ein Axiom vernünftiger politischer Ethik. ${ }^{8}$

Er stellte im Folgenden die Verbindung zum Weltparlament von 1893 her und erläuterte die Gründe für eine Fortsetzung des dadurch ausgelösten Prozesses:

Noch bemerkenswerter in seinem geistigen Gesichtskreis und Einfluss war das Parlament der Religionen bei der Welt-Ausstellung in Chicago 1893, welches siebzehn Tage dauerte und Teilnahme einer großen Anzahl von Delegierten erfuhr $[\ldots]$

Dieselbe Überzeugung und geschwisterlicher Impuls haben zur Organisation des Internationalen Rates unitarischer und anderer liberal-religiöser Denker und Wirkenden geführt. Man glaubt, dass seine Sitzungen, abgehalten alle zwei oder drei Jahre in verschiedenen Ländern, öffentliche Aufmerksamkeit anziehen und ihrem vereinten Zeugnis für fortgeschrittene religiöse und ethische Ideen Einfluss verleihen werden. Man glaubt weiterhin, dass es die Herzen und Hände einsamer, für religiöse Wahrheit und Freiheit Wirkender in vielen Ländern stärken wird und dass die religiösen Körperschaften, die damit verbunden sein mögen, sich selber anwachsend fühlen in Kraft und Einfluss durch das Bewusstsein größerer geschwisterlicher Beziehungen und eines breiteren organischen Lebens. In dieser guten Hoffnung treffen wir uns heute unter diesen glücklichen Vorzeichen um einzuweihen, worauf wir vertrauen, dass es eine permanente und einflussreiche Bewegung für die Einigung in allen Ländern und unter allen Menschen reiner Religion und vollkommener Freiheit werden wird. ${ }^{9}$

${ }^{8}$ Liberal Religious Thought at the Beginning of the twentieth Century; Addresses and Papers at the International Council of Unitarian and other Liberal Religious Thinkers and Workers, held in London, May (25.05.-03.06.) 1901. Hg. von William Copeland Bowie. London 1901, S. 324 u. $327 f$.

${ }^{9}$ Ebd., S. 329. 


\section{Weltkongresse in Amsterdam 1903 und Genf 1905}

Beim zweiten Weltkongress, der 1903 in Amsterdam stattfand, dachte Wendte in seinem Bericht bereits laut über die Organisation von Weltkongressen dieser Art in Asien nach. ${ }^{10}$

In seinem Bericht beim Genfer Weltkongress 1905 finden wir ein Gedicht ohne Quellenangabe, vermutlich von ihm selber. Ich habe versucht, es zu übersetzen, da es offenkundig seine Gedanken in poetischer Form abkürzt:

Wir mögen nicht denken unserer Väter Gedanken,

Ihre Glaubensbekenntnisse mögen unsere Lippen ändern,

Aber in dem Glauben, den sie teuer erkauften,

Werden unsere Herzen niemals wanken,

's war Glauben an Mensch, 's war Glauben an Gott,

's war Glauben an Wahrheit und Schönheit:

An Verstandes Anrecht und Freiheits Macht,

Und alles überprüfende Pflicht. ${ }^{11}$

Direkt nach diesem Gedicht beleuchtete Wendte die kritische Lage der religiösen Liberalen wie folgt:

Soweit wir die religiöse Situation rund um die Welt überblicken, finden wir, dass, während es überall eine gewaltige Bedeutung von latentem, unorganisiertem Liberalismus gibt, die Repräsentanten bewussten, vernünftigen und ehrerbietigen Freidenkens überall in einer Minderheit sind. Auch ist überall diese liberale Minderheit engagiert in einem entschlossenen Ringen, um die Religion des Geistes gegen eine aggressive Orthodoxie und reaktionäre Kräfte in Kirche und Staat aufrecht zu erhalten. Die Kräfte moderner Wissenschaft und Gelehrsamkeit fechten auf unserer Seite, das konstante Wachstum politischer Freiheit und sozialer Gerechtigkeit üben einen großen Einfluss zu unseren Gunsten aus, [...] Groß ist deshalb unser Bedarf, konfrontiert mit solchen Widersachern, unsere zerstreuten Kräfte zu ermutigen und einander zu stärken, damit wir nicht entmutigt und überwältigt werden im Ringen um religiöse Freiheit. [...] Wir müssen einander jene Sympathie und Kooperation erweisen, die allein unsere individuelle Sicherheit absichern können und den letztlichen und allgemeinen Triumph unserer Sache.

10 Religion and Liberty; Addresses and Papers at the second International Council of Unitarian and other Liberal Thinkers and Workers, beld at Amsterdam, September (01.-04.09.) 1903. Hg. von Pieter Herman Hugenholtz jr. Leiden 1904, S. 67.

11 Actes du 3 me Congrès International du Christianisme Libéral et Progressif, Genève (28.-31.08.) 1905. Hg. von Edouard Montet. Genf 1906, S. 14. 
Fünf Jahre später, in Berlin, fügte Wendte noch über den Genfer Kongress hinzu:

Daß die Stadt und Kirche Calvins unter den anderen Delegierten auch den geistigen Nachkommen von Servet und Socinus, den Unitariern, diese Gastfreundschaft zu Teil werden ließ, das war wirklich ein leuchtendes Zeichen religiösen Fortschrittes, ein Triumph christlichen Geistes über Kirchensatzungen und über die Vorurteile einer vergangenen Zeit. ${ }^{12}$

\section{Wendtes „Heimspiel“ beim Bostoner Weltkongress 1907}

Der Weltkongress in Boston 1907 war Wendtes Heimspiel, und er übernahm selber zusätzlich die Rolle des nationalen Generalsekretärs. Bei den Weltkongressen in anderen Ländern standen ihm stets Generalsekretäre der nationalen Vorbereitungs-Komitees zur Seite. 1910 in Berlin war dies der evangelische Pfarrer Dr. Gustav Adolf Fobbe. Mittlerweile war es mir möglich, eine Biografie unseres leider fast vergessenen nationalen Generalsekretärs zu erarbeiten. ${ }^{13}$

Beim Bostoner Kongress schwärmte Wendte regelrecht von den erreichten Erfolgen:

Was immer sonst dieser internationale Rat ausgeführt haben mag: er hat sicherlich die Fürsprecher religiöser Freiheit in vielen Ländern in engere Berührung und Bekanntschaft mit jedem anderen gebracht. Wir sind nicht mehr Fremde: wir sind Freunde. Wir drücken jedes anderen Hände heute mit einer Wärme, hervorgebracht durch Vertrauen und Dankbarkeit; wir schauen in aller anderen Gesichter mit herzlichem / liebevollem Interesse; wir hören jedes anderen Worte mit eifriger Erwartung. Wir werden jedem anderen von unseren individuellen Erfahrungen erzählen seit wir uns zuletzt trafen - unsere Versuche und Niederlagen, unsere Triumphe und Gewinne, unsere unvergänglichen Treuhandschaften und Hoffnungen $[\ldots]{ }^{14}$

Er begrüßte

das Herauskommen mehrerer verschiedener Reihen populärer Handbücher über theologische, philosophische und religiöse Themen in Deutschland und anderen Ländern, geschrieben von hervorragenden Gelehrten, welche sich schon weiter Verbreitung erfreuen, - ein erfreulicher Beweis, dass die

\footnotetext{
12 Fünfter Weltkongress für freies Christentum und religiösen Fortschritt, Berlin 5. bis 10. August 1910. Protokoll der Verhandlungen. Hg. von Max Fischer und Friedrich Michael Schiele. Berlin 1910, S. 85.

13 Michael Sturm-Berger: Pfarrer Dr. Gustav A. Fobbe und das interreligiöse „Gebeimnis" am Gesundbrunnen. Eine Biographie anlässlich seines 65. Todestages am 09.02.2012. Erfurt 2012.

${ }^{14}$ Freedom and Fellowship in Religion; Proceedings and Papers of the fourth International Congress of Free Christians and other Religious Liberals, beld at Boston. U.S.A., Boston September 22-27, 1907. Hg. von Charles William Wendte. Boston, Mass. 1907, S. 55.
} 
Schlussfolgerungen moderner historischer und kritischer Wissenschaft nicht länger der exklusive Besitz der gebildeten Klassen und der Universität sind, sondern sie zunehmend zur Kenntnis und Fassungskraft der schlichten Gottesleute zu bringen sind. Wenn dies erfolgreich vollendet werden sollte, wird eine religiöse Revolution oder eher Transformation nicht weit entfernt sein. ${ }^{15}$

„Der wahre Liberale spricht nicht nur die Wahrheit, sondern er spricht sie in Liebe. Er toleriert nicht nur, er liebt seine Mitmenschen. "16 Diese beiden bedeutungsvollen Sätze fassen auch einen der wenigen ins Deutsche übersetzten Artikel Wendtes schön zusammen: $W$ as heißt es religiös liberal sein? Ein unitarischer Traktat. ${ }^{17}$

Wir bemerken, denke ich, dass man viel Substanzielles aus seinen Texten ablesen kann - und sind damit wieder beim Berliner Weltkongress angekommen.

\section{Nochmals zum Berliner Weltkongress 1910}

Wendte hatte bereits 1908 einen Nachruf auf den verstorbenen Berliner Professor Otto Pfleiderer verfasst und gab beim Kongress nochmals seiner großen Wertschätzung Ausdruck:

Sein liebevolles Entgegenkommen und der anregende Verkehr mit ihm in Berlin gehörten für uns Ausländer zu den schönsten Vorerinnerungen unserer gegenwärtigen Zusammenkunft. [...] Er war und ist unser aller Lehrer und Meister und durch sein anregendes und biederes Wesen uns als guter, edler Mensch und treuer Mitarbeiter eine unvergeßliche Erinnerung. ${ }^{18}$

Der nationale Generalsekretär des Berliner Kongresses, der bereits erwähnte Pfarrer Dr. Fobbe, war übrigens ein begeisterter Student Professor Pfleiderers gewesen.

In seiner englischsprachigen Zusammenfassung über den Berliner Weltkongress schrieb Wendte:

einer intelligenteren und verständnisvolleren Zuhörerschaft hat niemand jemals ins Angesicht gesehen. [...] Sehr eindrucksvoll war auch die vorherrschende Offengesinntheit, Geduld und Höflichkeit, gezeigt durch die Zuhörerschaft gegenüber diesen Rednern und durch die Redner untereinander.

\footnotetext{
15 Ebd., S. 58.

16 Ebd., S. 64.

17 Charles Willliam Wendte: Was heißt es religiös liberal sein? Ein unitarischer Traktat. In: Die Christliche Welt. 21. Jg., Nr. 37, 12.9.1907, Sp. 881-884.

18 Fischer/Schiele: Fünfter Weltkongress, S. 90.
} 
[...] Niemals sind so viele divergente Gesichtspunkte bei einem unserer Kongresse präsentiert worden. ${ }^{19}$

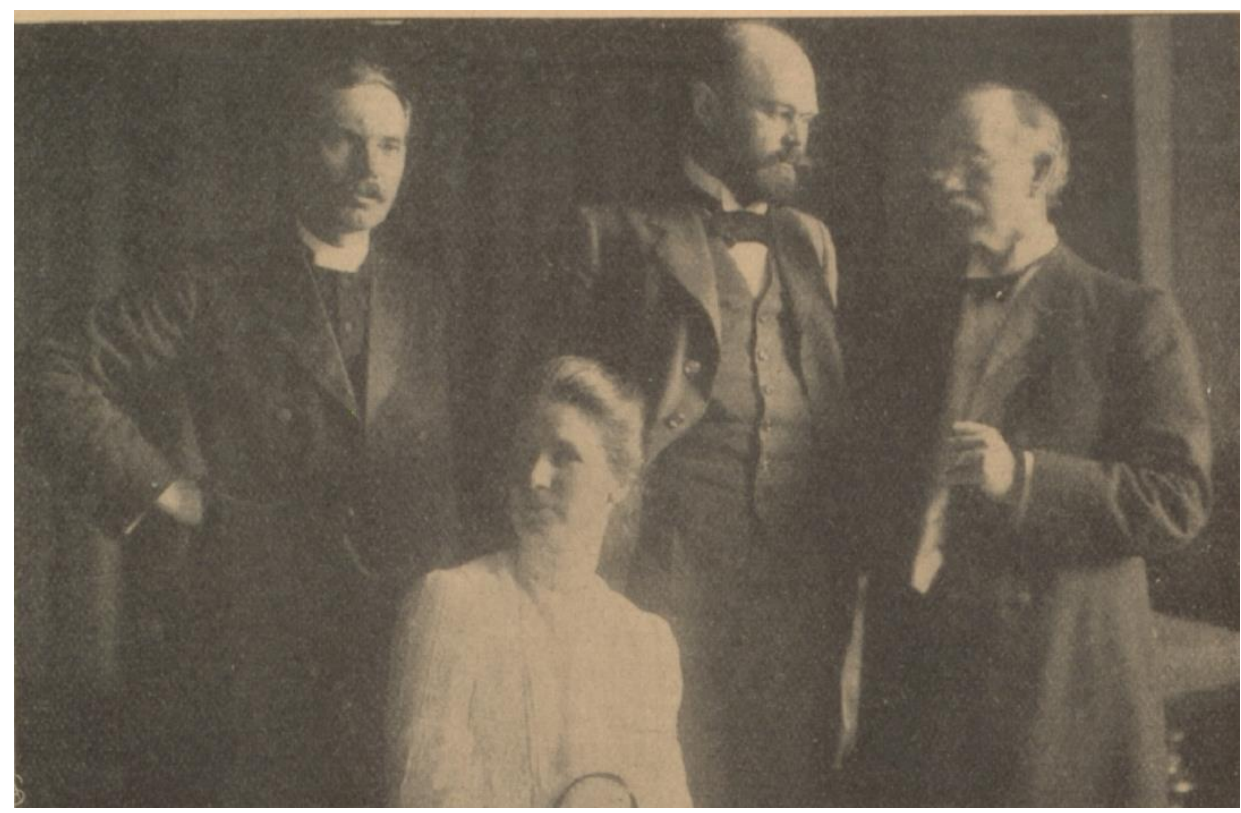

Abbildung 3: Beim Weltkongress 1910 in Berlin (v.l.n.r.): Rev. Tudor Jones (Australien), Ehepaar Dr. Ada und Prof. Heinrich Weinel (Jena), Wendte (aus: Die Woche, Berlin, 12. Jg., Nr. 33, 13.8.1910, S. 1382)

Leider musste er später hinzufügen:

Kurz nach dem Kongress, möglicherweise durch ihn dazu veranlasst, hielt der Kronprinz von Preußen eine Ansprache, in welcher er das Wachstum internationaler Ansichten in Deutschland stark missbilligte. Dass unter solchen Bedingungen der Berliner Kongress so weitherzige Gastfreundschaft $\mathrm{zu}$ ausländischen Nationen und fremden Ideen entfaltete, war ein moralischer Triumph der ersten Ordnung. ${ }^{20}$

19 Fifth International Congress of Free Christianity and Religious Progress Berlin August 5-10, 1910; Proceedings and Papers „Einheit durch Freibeit". Hg. von C. W. Wendte und Valentine David Davis. Berlin/London 1911, S. 4.

20 Wendte: Fellowship, Bd. 2, S. 366. 


\section{Der Pariser Weltkongress 1913 und die Entwicklung danach}

Doch hatte sich auch organisatorische Kritik an den über 150 innerhalb einer Woche abgehaltenen Ansprachen ergeben. In seiner englischsprachigen Zusammenfassung von 1913 über den Weltkongress in Paris fand diese wie folgt ihren Ausdruck:

Man macht geltend, und mit Recht, dass diese Treffen zu oft sind, die diskutierten Themen zu vielseitig; dass ihre Programme zu überfrachtet sind mit Rednern, so dass sie eine hastige und oberflächliche Behandlung der präsentierten Gegenstände, Abschneiden der freien Debatte haben und indem sie in einer intellektuellen Übersättigung resultieren, welche den vollen Nutzen unmöglich macht, der gezogen werden kann aus den Ansprachen, ob gelehrt oder geschickt, und verhindert oft jene praktische Betrachtung und Aktion, welche eines der Merkmale dieser Treffen sein sollte. ${ }^{21}$

Ein sehr bemerkenswertes, in Paris mit Hilfe von Vorträgen kontrovers diskutiertes Thema war: Ist eine universale Religion möglich oder wünschenswert?

Nun war es gerade dieser Kongress, bei dem erstmals ein gelehrtes Mitglied der Bahá'i-Weltgemeinde sprach. Es war Hippolyte Dreyfus, welcher vom damaligen Oberhaupt dieser Gemeinde, Abdu'l-Bahá (1844-1921), dazu beauftragt worden war.

Letzterer war mit Wendte befreundet und hatte ihn 1912 in Boston besucht. Für Oktober 1914 bis März 1915 hatte Wendte mit ihm und anderen zusammen eine „weltweite Pilgerreise religiös Liberaler“ geplant. Diese konnte wegen Ausbruch des Ersten Weltkrieges genauso wenig stattfinden, wie der für 1916 in London angedachte Weltkongress.

Erst 1920 gelang es wieder, eine etwas bescheidenere Versammlung zum 300. Jubiläum der Pilgerväter in Boston und Plymouth zu organisieren. Wendte hatte sich ununterbrochen bemüht, briefliche Kontakte zu den Mitarbeiterinnen und Mitarbeitern der Weltkongresse zu halten. Er pflegte auch freundschaftliche Kontakte zu Mitgliedern zahlreicher christlicher Konfessionen, Sikhs, Parsen, Buddhisten, Anhängern japanischer und chinesischer Religionsgemeinschaften. Nach dem Krieg trat er für eine in Großbritannien gegründete Liga der Religionen ein und erwähnte den von Rudolf Otto in Deutschland konzipierten Religiösen Menschbeitsbund. Anschließend kündigte er an, dass er zwar zeitlebens der Weltkongress-Bewegung verbunden bleiben, aber aus Altersgründen zurücktreten werde - und weil er glaube, dass jetzt die Leitung der Weltkongresse nach Europa verlegt werden sollte. Daher übergab er mit Zustimmung des Rates sein Amt an Rev. William H.

${ }^{21}$ Charles Wilhelm Wendte: Religious Liberals in Council; an Appreciation of the sixth International Congress of Religious Progress; Paris, France July 16-22, 1913; (Reprinted from the Christian Register, Boston) 1913, S. 4 . 
Drummond in London. ${ }^{22}$ In der Folgezeit schrieb er weiter an seiner Autobiografie, aus der ich oben mehrfach zitierte.

Am 9. September 1931 starb er in Berkeley (Kalifornien), im Alter von 87 Jahren. Seine Korrespondenzen und wissenschaftlichen Nachlässe werden in der Theologischen Andover-Harvard-Bibliothek in Cambridge (Massachusetts) aufbewahrt. Ein halbwegs vollständiges Verzeichnis seiner Veröffentlichungen existiert bis heute meines Wissens leider nicht.

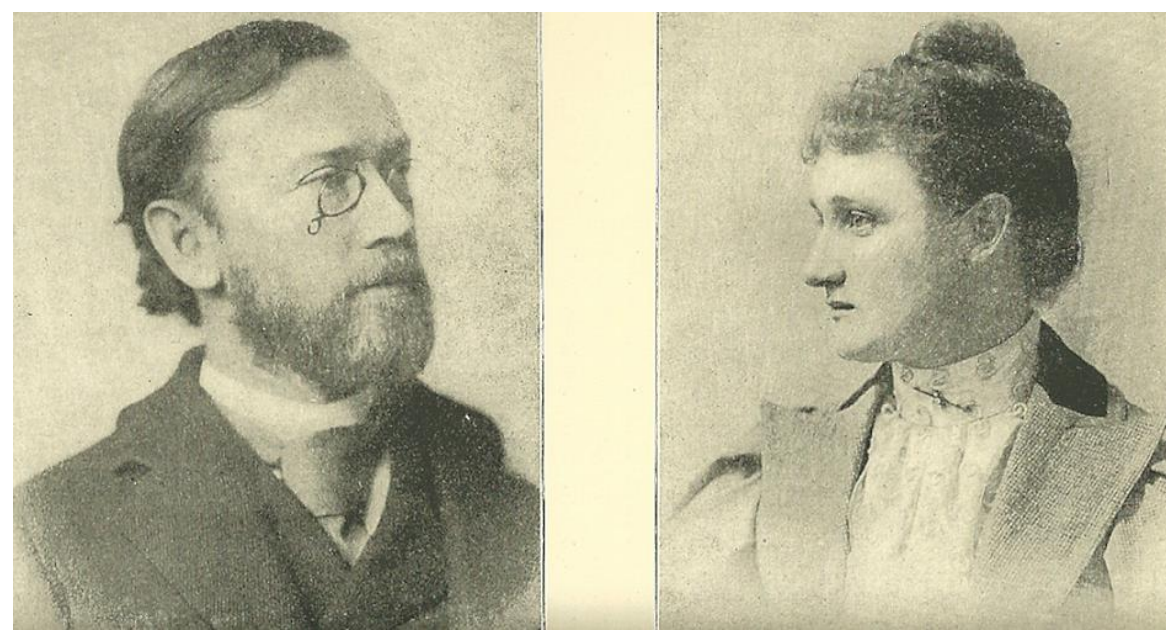

Abbildungen 4 und 5: Wendte und seine Gattin Abbie Louise, geb. Grant (aus: The Wider Fellowship, Bd. 2, S. 128)

\footnotetext{
22 New Pilgrimages of the Spirit; Proceedings and Papers of the Pilgrim Tercentenary Meeting of the International Congress of Free Christians and other Religious Liberals; held at Boston and Plymouth, U.S.A., October 3-7, 1920. Boston, Mass. 1921, S. 87-92 u. 151-154.
} 